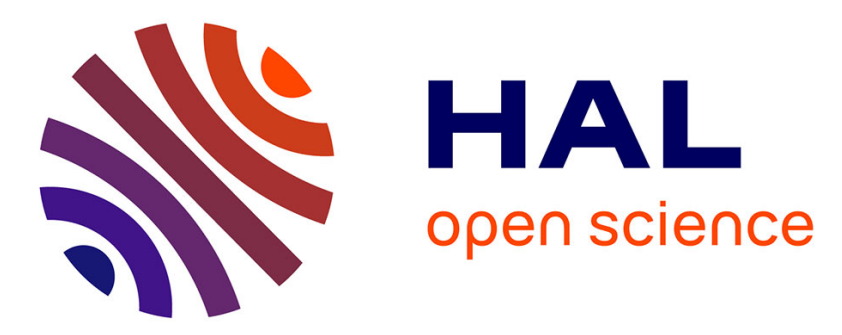

\title{
Role of interchain hopping on electron spin relaxation in quasi-one-dimensional case
}

\author{
M. Sanquer, S. Bouffard, L. Forro
}

\section{To cite this version:}

M. Sanquer, S. Bouffard, L. Forro. Role of interchain hopping on electron spin relaxation in quasi-one-dimensional case. Journal de Physique, 1986, 47 (6), pp.1035-1041. 10.1051/jphys:019860047060103500 . jpa-00210280

\section{HAL Id: jpa-00210280 https://hal.science/jpa-00210280}

Submitted on 1 Jan 1986

HAL is a multi-disciplinary open access archive for the deposit and dissemination of scientific research documents, whether they are published or not. The documents may come from teaching and research institutions in France or abroad, or from public or private research centers.
L'archive ouverte pluridisciplinaire HAL, est destinée au dépôt et à la diffusion de documents scientifiques de niveau recherche, publiés ou non, émanant des établissements d'enseignement et de recherche français ou étrangers, des laboratoires publics ou privés. 


\title{
Role of interchain hopping on electron spin relaxation in quasi-one-dimensional case
}

\author{
M. Sanquer, S. Bouffard and L. Forro $\left({ }^{+}\right)$ \\ IRDI-DMECN-DTech/SESI, Centre d'Etudes Nucléaires, 92265 Fontenay aux Roses Cedex, France \\ $\left({ }^{+}\right)$Institute of Physics of the University, Bijeniéka Cesta 46, P.O.B. 304, 41001 Zagreb, Yugoslavia
}

(Reçu le 4 septembre 1985, révisé le 27 décembre 1985, accepté le 30 janvier 1986)

\begin{abstract}
Résumé. - Devant les difficultés théoriques pour prévoir la valeur et la variation en température de la largeur de raie de résonance paramagnétique électronique des conducteurs organiques, la plupart des expériences ont été réalisées sur des familles de composés. Afin de réduire le nombre de paramètres changeant lors de ces expériences, nous avons modifié dans le même échantillon (TTF-TCNQ) le couplage interchaîne en introduisant des coupures dans les chaînes conductrices. Nous observons un rétrécissement inhabituel de la raie de résonance lorsque le désordre augmente. Cette variation de la largeur de raie peut être décrite par un modèle de spins localisés sur des segments métalliques en associant le renversement des spins aux sauts intersegments. Les paramètres introduits dans ce modèle sont en accord avec ceux déterminés lors de mesures de conductivités électriques. Il est cependant nécessaire d'introduire une interaction dipolaire entre spins localisés sur des segments adjacents pour décrire tout le domaine de désordre. Ce dernier terme est dominant à basse température et pour les forts degrés de désordre. Ces résultats démontrés dans le cas de TTF-TCNQ peuvent être étendus à l'ensemble des conducteurs organiques.

Abstract. - Theories cannot actually predict the value and the $T$-dependence of the electronic spin resonance linewidth of organic conductors. To compensate this difficulty, most of the experiments have been carried out on families of compounds. In order to reduce the number of parameters, we have modified on the same compound (TTF-TCNQ) the interchain coupling by introducing chain breakings. We observed an unusual decrease of the linewidth when the degree of disorder increases. A model of spins localized on metallic segments, and where the spins are reversed by the interchain jumps, described this variation of the linewidth. The parameters found in this model are in agreement with those determined by conductivity measurements. However, in order to take into account the whole disorder range, the dipolar interaction between localized spins should not be neglected : this term is dominant at low temperatures and for the highest disordered compounds. One can generalize these results which are demonstrated on the case of TTF-TCNQ to the other organic conductors.
\end{abstract}

\section{Introduction.}

In spite of a nice electron spin resonance line (ESR) observed in one-dimensional compounds, ESR studies are often limited to phenomenological considerations because of the lack of any theoretical model describing the spin relaxation in quasi-one-dimensional conditions. Nevertheless experimental studies of families of compounds [1-3] or alloys [4-5] give some general results on the spin relaxation. First the linewidth is closely dependent on the dimensionality of the compound : when the anisotropy increases, the ESR line is narrowed [4]. Second the linewidth varies like $(\Delta g)^{2}$ where $\Delta g$ is characteristic of the spin-orbit coupling, which is itself essentially related to the molecular composition.
However, these approaches have the great disadvantage to need to determine what is related to the variation of the spin relaxation rate (or of the dimensionality) and what is introduced by the intrinsic properties of the molecules. In this paper, we demonstrate that irradiation experiments allow a more straightforward study.

Moreover the temperature dependence of the spin relaxation rate is the least understood problem; indeed, in the metallic regime, the linewidth decreases on heating in TTF-TCNQ [1], or increases in (TMTSF) $)_{2}-\mathrm{X}$ [6] or even presents a minimum for TMTSF-DMTCNQ [1]. For this point irradiation also gives new results on the respective roles of interchain jump frequency and electron-phonon scattering time. 
The paper is arranged as follows : after an experimental section, we discuss the low defect concentration behaviour in connection with the electron transverse motion in the case of TTF-TCNQ. The next section is a discussion of the higher doses when the electrons are localized on segments. A comparison with the $\mathrm{Qn}(\mathrm{TCNQ})_{2}$ behaviour is then possible. The susceptibility of irradiated compounds and the determination of the defect concentration are reported in reference [7].

\section{Experimental section.}

The ESR spectra were obtained by using a conventional Bruker model ER 200 D spectrometer. The klystron frequency $(\sim 9.3 \mathrm{GHz})$ was measured with a Systron model $6245 \mathrm{~A}$ frequency counter and the static field by a Bruker ER 035 M NMR gaussmeter. The samples were attached with a teflon film on a quartz holder inside a standard Oxford Instrument continuous flow helium system. This apparatus uses an iron-gold chromel thermocouple which was found to be accurate to within $1 \mathrm{~K}$ below $20 \mathrm{~K}$. At higher temperatures a copper-constantan thermocouple placed near the sample was used to measure the sample temperature. Generally the observed lines have a Lorentzian shape and the susceptibility is obtained as the product $A \times(\Delta H)^{2}$ where $A$ is the amplitude and $\Delta H$ the peak-to-peak linewidth. The absolute magnitude was measured by direct comparison with the susceptibility of a copper sulfate sample. When the sample orientation is not mentioned, it is as follows : the static field is applied along the $c^{*}$-axis and the radiofrequency field along the conducting axis.

Irradiations were carried out either with the $2.5 \mathrm{MeV}$ Van de Graaff or with the $100 \mathrm{keV}$ electron accelerator of Fontenay-aux-Roses. The irradiation temperatures were either $21 \mathrm{~K}$ or room temperature.

At low temperatures the susceptibility of irradiated TTF-TCNQ shows an upturn that follows an $A T^{-\alpha}$ dependence with $A$ increasing roughly linearly with the defect concentration. By comparison with a Curietail we can estimate an approximate number of spins created by irradiation [8]. The spin concentration thus determined is larger by a factor of 3 or 5 than the defect concentration estimated by conductivity measurements, using the mixing rate of transverse and longitudinal resistances at room temperature [9].

Afterwards all the defect concentrations mentioned in this paper are conductivity defect concentrations with an average absorbed energy of $50 \mathrm{MGy}$ necessary to create $1 \%$ of defects per mole $(1 \mathrm{~Gy}=100 \mathrm{rads}=$ 1 joule $\mathrm{kg}^{-1}$ ).

\section{Experimental results.}

In this work we have focused our attention only on the irradiation doses which are sufficient to completely smear out the phase transition. The lower concentrations have been already studied by Korin-Hamzic et al. [8] in TTF-TCNQ.
The variation of the linewidth with the irradiation dose is presented for different compounds in figure 1.

Figure 2 shows the linewidth as a function of temperature for TTF-TCNQ containing different amounts of defects. The main result is the unusual decrease of the linewidth in the metallic range when the degree of disorder increases. Indeed this decrease is not the expected result considering the behaviour of the conduction electron spin resonance (CESR) in isotropic metals [10] or in materials with a lower dimensionality such as intercalated graphite [11]. In these compounds the linewidth has been found to be proportional to the resistivity, so it increases with the degree of disorder.

\section{Discussion.}

4.1 LOW DEFECT CONCENTRATION BEHAVIOUR. - The convenience of ESR studies on organic metals is somewhat surprising for it is well known that the conduction electrons of only a few metals give rise to an observable resonance line. The theories describing the ESR of isotropic conductors have been first developed by Overhauser [12], Elliott [13] and Yafet [14]. The dominant process leading to the reversal of the electron-spin is a spin-lattice relaxation, which comes from the modulation of the spinorbit interaction by acoustical phonons. Moreover Elliott has pointed out the relation between CESR

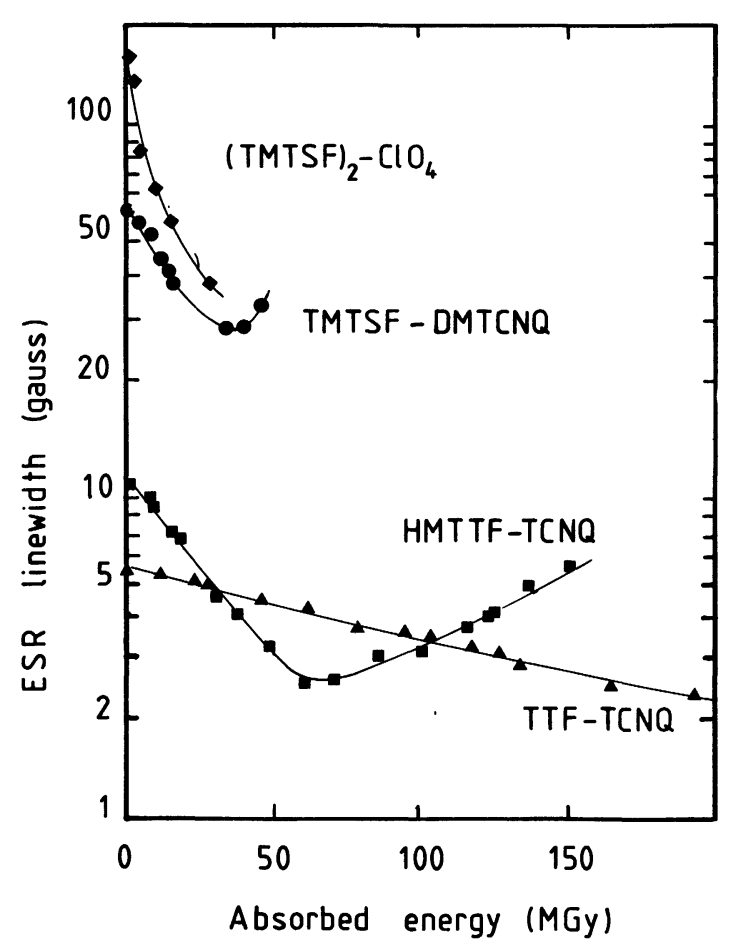

Fig. 1. - Dependence of the linewidth as a function of the absorbed energy (irradiation dose) for various metallic organic compounds. The results, except for (TMTSF) ${ }_{2}$ $\mathrm{ClO}_{4}(150 \mathrm{~K})$, were obtained at room temperature. 


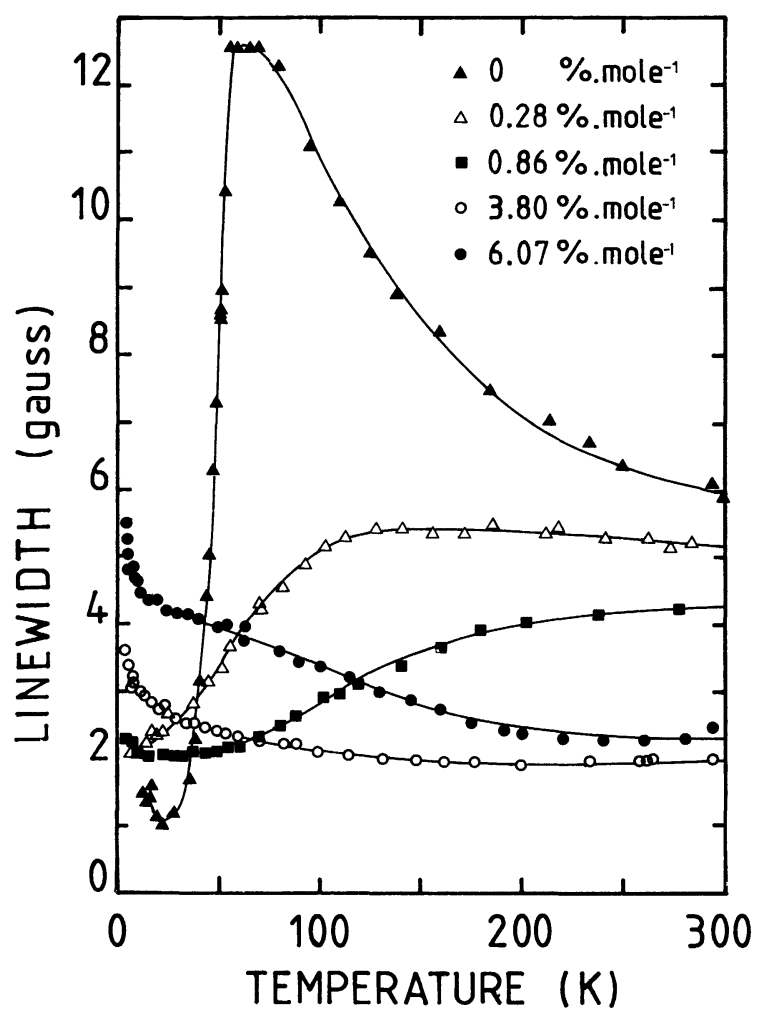

Fig. 2. - Temperature dependence of the linewidth of pure and irradiated TTF-TCNQ. One has to mention the decrease of the linewidth at high temperatures when the defect concentration increases.

linewidth $(\Delta H)$, spin-orbit interaction $(\Delta g)$ and electron-phonon scattering time $(\tau)$, namely :

$$
\Delta H \simeq \frac{(\Delta g)^{2}}{\tau} .
$$

The validity of Elliott's relation has been experimentally verified in metals at low temperatures [10] and also in lithium intercalated graphite compounds [11]. However Elliott's relation fails to describe the ESR linewidth of organic metals : by introducing the observed relaxation times and $g$-shift in relation (1), one obtains linewidths one to three orders of magnitude larger than those measured. The remarkably narrow CESR line of most of the quasi-one-dimensional organic conductors has been discussed by several authors $[4,6,15]$. The reason for the failure of Elliott's relation is the inefficiency of the spin-orbit coupling to induce electronic spin reversal for strictly one-dimensional electron. Thus only the departure from one-dimensionality could explain the electron spin relaxation. Weger [16] has suggested that the deviation from the originally flat Fermi surface can be taken into account by introducing the ratio $\tau_{\|} / \tau_{\perp}$, where $\tau_{\|}$is the scattering time for the electronic motion along the chain and $\tau_{\perp}$ the average time spent by an electron on a given chain [17]. Relation (1) becomes :

$$
\Delta H \simeq \frac{(\Delta g)^{2}}{\tau_{\|}} \frac{\tau_{\|}}{\tau_{\perp}}=\frac{(\Delta g)^{2}}{\tau_{\perp}}
$$

This relation may be also crudely understood by considering that only interchain jumps with a frequency $\tau_{\perp}^{-1}$ are able to reverse the spin via the spinorbit coupling.

This model describes pretty well the case of TTFTCNQ for which, at least above $100 \mathrm{~K}$, the transverse conductivity is diffusive and should be written $\sigma_{\perp}=$ $N\left(E_{\mathrm{F}}\right) e^{2} b^{2} \tau_{\perp}^{-1}$ where $b$ is the interchain distance $[14,17]$; the linewidth and the transverse conductivity, which both increase on cooling, should therefore behave similarly whatever the reason for the change of $\tau_{\perp}^{-1}$. This is in fact roughly true for pressure and temperature dependences $[14,17]$. But the observed decreasing linewidth on cooling in other organic conductors, (TMTSF) ${ }_{2} \mathrm{ClO}_{4}$ for example, suggests that formula (2) is invalidated in that case. Bloch [18] proposes the following correction to Elliott's relation (1) :

$$
\Delta H=\frac{(\Delta g)^{2}}{\tau_{\|}} \cdot\left(\frac{t_{\perp}}{t_{\|}}\right)^{2}
$$

where $\tau_{\|}$is an electron-phonon relaxation-time which increases on cooling. One has to mention that this qualitative relation is not supported by any experimental investigation.

It is also possible to include the case of TTF-TCNQ and related compounds in the following picture : Tomkiewicz [4] puts forward the correlation between the existence of a giant Kohn anomaly developing in the phonon spectrum at $2 k_{\mathrm{F}}$ in the metallic regime and the fact that the linewidth increases with decreasing temperature. She connects the upturn of the linewidth with the increasing number of $2 k_{\mathrm{F}}$ phonons able to relax electron spins (if they are not strictly one-dimensional). This latter argument has been also used in a comparative ESR study of three BEDT-TTF salts [3]. However in that case, there is no relation between linewidth and transverse conductivity except casual.

We propose a single representation of the two former approaches :

$$
\Delta H=A(\Delta g)^{2} \tau_{\perp}^{-1}+B(\Delta g)^{2} \tau_{\|}^{-1}
$$

which could describe intermediate cases, following the respective values of the prefactors $A$ and $B$.

In a two-dimensional case, i.e. when the transverse conductivity is coherent, interchain jump frequency has no sense and $A$ is zero : the electron-phonon scattering time governs the spin relaxation.

On the contrary, in a strictly one-dimensional case, the electron-phonon relaxation is inefficient to reverse spins $(B=0)$ and $\tau_{\perp}^{-1}$ vanishes. In the quasi-onedimensional case, $B / A$ depends on the dimensionality of the considered compound. We will show that irradiation decreases $B$ (with respect to $A$ ) and acts like an increase of anisotropy.

In fact, irradiation is a manner to reduce the interchain motion. The infinite conducting chains are 
transformed by irradiation in an assembly of metallic segments, bounded by strong defects. In such a framework of metallic segments, longitudinal and transverse conductivities are dominated by the transverse hopping of electrons between neighbouring segments. As a consequence of the difference between the adjacent segment lengths, the different energy separations induce a phonon assisted hopping between neighbouring segments [19]. Therefore the transverse hopping frequency can be written [20] :

$$
\tau_{\perp}^{-1}(c)=\tau_{\perp}^{-1}(0) \exp \left(\frac{-\varepsilon}{k T n}\right)
$$

where $\varepsilon$ is the bandwidth and $\bar{n}$ the average number of sites in a segment $\left(\frac{1}{\bar{n}}=c\right.$ is the defect concentration). Accordingly, the transverse conductivity and the ESR linewidth are given by :

$$
\begin{gathered}
\sigma_{\perp}(c)=\sigma_{\perp}(0) \exp \left(\frac{-\varepsilon c}{k T}\right) \\
\Delta H \simeq(\Delta g)^{2} \tau_{\perp}(0)^{-1} \exp \left(\frac{-\varepsilon c}{k T}\right) .
\end{gathered}
$$

The $g$-tensor does not depend on the defect concentration (at low doses) and the symmetry of the linewidth tensor is also unaffected by the presence of defects [21]. So, surprisingly, the linewidth should decrease exponentially when the defect concentration increases.

The linewidth narrowing corresponds to an electronic localization on shorter and shorter metallic particles when their size decreases [22].

Figure 3 shows the linewidth of TTF-TCNQ as a function of the defect concentration at different temperatures in the metallic regime. The solid lines represent the best fits with the following relation :

$$
\Delta H=\Delta H_{0} \exp \frac{-\varepsilon c}{k T}+c \alpha+\beta .
$$

The linear term due to the dipolar interaction between localized spins, which is dominant at high doses, will be discussed in the next section. Table I gives the parameters of equation (8) for various temperatures. The $\alpha$ and $\beta$ coefficients are essentially given by the linear part at high defect concentrations. The $\varepsilon$ coefficient is determined by the room temperature fit and kept independent of $T$ and $c$ in accordance with the interrupted strand model where $\varepsilon$ is the bandwidth. The value $1.2 \mathrm{eV}$ for $\varepsilon$ is the same as that determined by the variation of the transverse conductivity with irradiation dose [7]. Expression (8) which fits the linewidth over a very large defect concentration range in all the metallic regime confirms that the interrupt strand model is appropriate to interpret the magnetic properties of the disordered organic conductors.

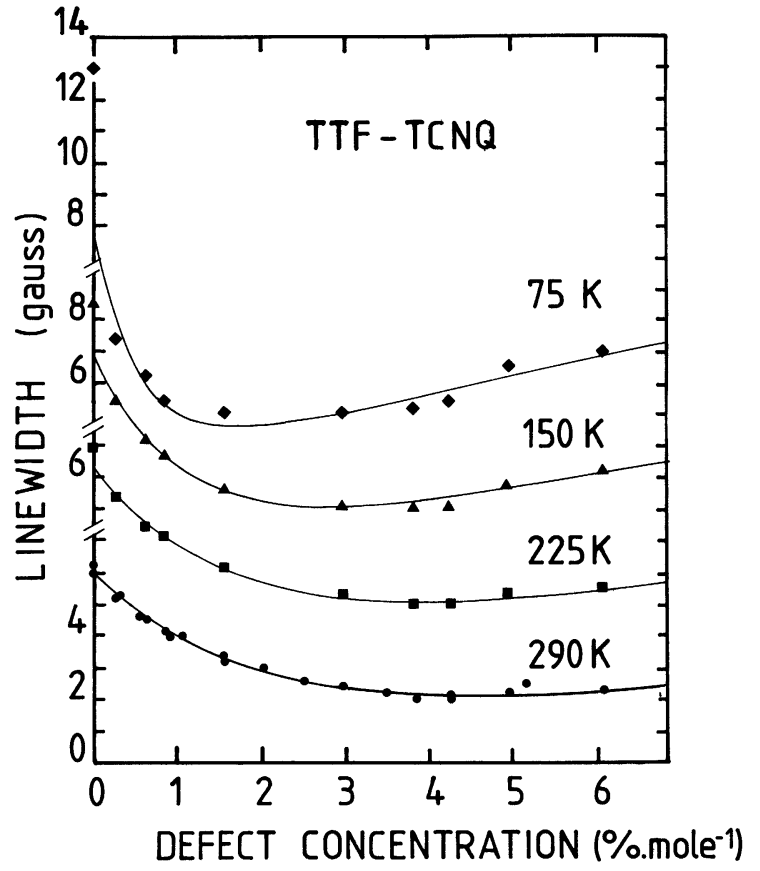

Fig. 3. - Defect concentration dependence of the linewidth of TTF-TCNQ at constant temperature (for clarity the curves are shifted). The lines represent the best fit with the model developed in the text. Note the discrepancy at low temperatures between the fit and the experimental points for the pure case $(c=0)$.

Table I. - Parameters of equation (8) used to fit the experimental curves of figure 2.

\begin{tabular}{|c|c|c|c|c|}
\hline$T(\mathrm{~K})$ & $\Delta H_{0}(\mathrm{G})$ & $\varepsilon(\mathrm{K})$ & $\alpha(\mathrm{G})$ & $\beta(\mathrm{G})$ \\
\hline 290 & 6.0 & 14100 & 32.11 & 0.0 \\
\hline 225 & 6.4 & 14100 & 32.11 & 0.2 \\
\hline 150 & 7.0 & 14100 & 44.93 & 0.3 \\
\hline 75 & 8.0 & 14100 & 57.95 & 0.35 \\
\hline 50 & - & - & 57.95 & 0.35 \\
\hline
\end{tabular}

However a deviation between fits and pure TTFTCNQ linewidth emerges when approaching the Peierls transition. At $75 \mathrm{~K}$ for instance the measured linewidth is $13 \mathrm{G}$ (and sometimes more for some batches) instead of $8 \mathrm{G}$ for the fitted values (see Fig. 3). A deviation from the interrupted strand model at low irradiation doses is also seen in transverse conductivity measurements at $73 \mathrm{~K}$. In figure 4 we have plotted the normalized transverse conductivity and linewidth together with our fits based on the interrupted stand model (see Eqs. (6) and (7)). 


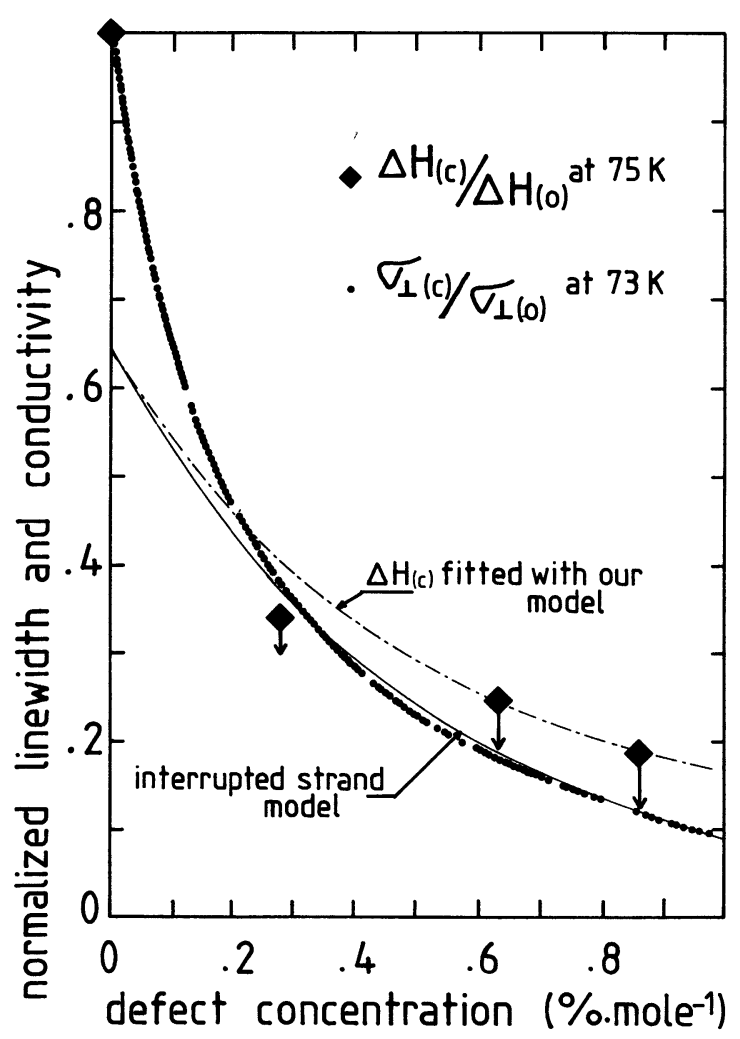

Fig. 4. - Transverse conductivity and linewidth normalized to their values for pure TTF-TCNQ as a function of the defect concentration. The arrows represents the subtraction of the dipolar contribution. The lines show the fits with the metallic segment model established from a defect concentration range ten times larger than that represented. Note the similarity between the conductivity and the linewidth dependence.

For conductivity, the parameters are entirely determined by the high defect concentration regime (not shown in the figure) : the slope gives $\varepsilon$ and the value of the conductivity imposes $\sigma_{\perp}(0)$. One can note the similarity between normalized transverse conductivity and linewidth curves, especially if the dipolar contribution is subtracted from the total linewidth. This confirms Weger's remark that the linewidth behaves like the transverse conductivity. But both properties decrease faster than predicted by the metallic segment model.

The only way to explain this deviation is to consider that, in pure or low damaged TTF-TCNQ below say $100-150 \mathrm{~K}$, the transverse integral $t_{\perp}$ is larger than the average spacing $\varepsilon c$ of the electronic energy levels within a segment. With respect to localization theories, the ratio $\varepsilon c / t_{\perp}$ is precisely adopted as the measure of the localization strength of the disorder [23].

Localization occurs when the energy mismatch between levels of adjoining segments exceeds the extend to which the transfer energy can shift them, making the alignment of levels impossible : $t_{\perp}<\varepsilon c$. For TTF-TCNQ, $\varepsilon c$ overcomes $t_{\perp}$ for $c$ higher than $0.4 \%$ mole $\left(t_{\perp} \simeq 5 \mathrm{meV}[17], \varepsilon \simeq 1.2 \mathrm{eV}\right)$. Figure 4 shows that upon this concentration the interrupted strand model fits the experimental points. One could notice that below this defect concentration the transverse resistance increases linearly with defect concentration. This suggests that there is no electronic localization process.

In this case, to explain the decrease of the linewidth we have rather to consider that defects decouple the conducting chains and reduce the dimensionality $(B / A$ decreases in relation (4)). The coherent transverse conductivity becomes diffusive, then relation (7) can be used again.

4.2 High DEFECT CONCENTRATION BEHAVIOUR. - The linear increase of the linewidth for the highest defect concentration implies another contribution to the spin relaxation. Before discussing the physical reasons which qualitatively explain this linear contribution to the linewidth, let us review the different ways expected to reverse the electronic spins in irradiated TTF-TCNQ. First one has to keep in mind that at high defect concentrations and at low temperatures, the electrons are strongly localized on segments : the resistivity is increasing by a factor larger than $10^{6}$ at $70 \mathrm{~K}$. As far as this localization is concerned, spins are sensitive to the dipolar interaction with the other electronic localized spins and to hyperfine interaction with the nuclear spins. Moreover the orientational spin motion induced by the exchange interaction could average the local fields produced by dipolar and hyperfine coupling. At low temperatures, when the intersegment jumps are prevented for the electronic spins, these interactions are expected to govern the linewidth, but when the temperature increases, the activated transverse hopping becomes more and more efficient.

This electronic translational motion also averages the dipolar and hyperfine interactions and overcomes the exchange when the electrons are highly mobile. At that time the spin-lattice relaxation via spin-orbit coupling becomes the leading process (the linewidth is then described by relation (2)). So when the temperature decreases or when the disorder increases the electrons spend more and more time on a given segment and the spin-lattice relaxation is progressively overcome by relaxation through the coupling between localized spins. To corroborate this decomposition, we have plotted the residual linewidth

$$
\Delta H_{1}=(\Delta H \text { observed })-\left(\Delta H_{0} \exp \frac{-\varepsilon c}{k T}\right)
$$

above $50 \mathrm{~K}$ and when the spins are localized in the disordered compound, the observed linewidth below $50 \mathrm{~K}$ (Fig. 5). The continuous variation in the whole temperature range allows us to assume that the residual linewidth is related to a relaxation involving localized electron spins on segments. Such an assumption has been already proposed for disordered Qn 


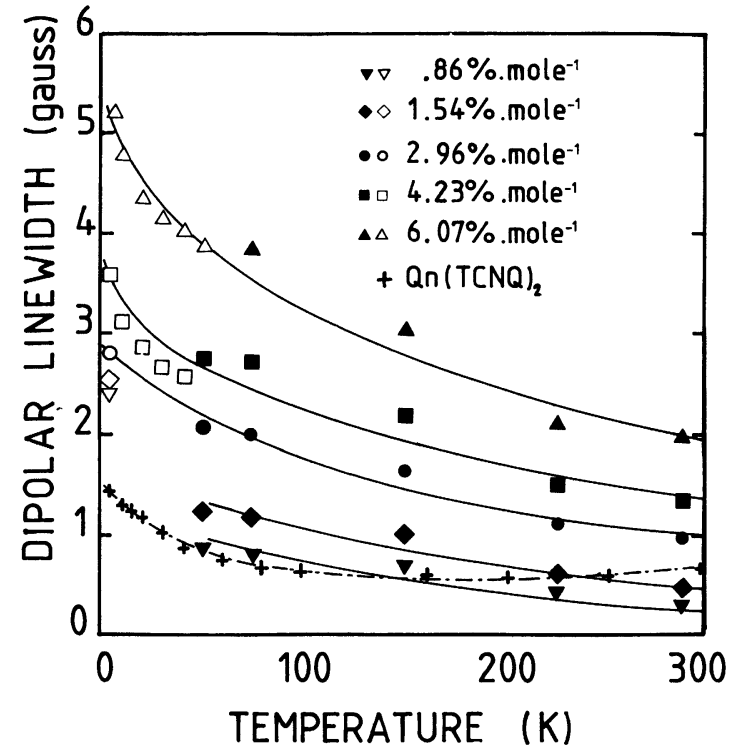

Fig. 5. - In this figure, the linewidth associated with the dipolar interaction as a function of temperature is represented. At low-temperature (opened signs) the linewidth is the measured one, when at higher temperature (closed signs) the reported linewidth is the linewidth calculated by subtraction of the Weger contribution (see text). For comparison the result for Qn(TCNQ) 2 [24] (crosses) showing the same pseudo-Curie tail than TTF-TCNQ containing $0.86 \%$ of defect per mole is reported.

$(\mathrm{TCNQ})_{2}$ [24], thus we can compare irradiated Qn(TCNQ) ${ }_{2}$ and TTF-TCNQ samples showing the same pseudo-Curie tail. Both the observed Qn(TCNQ) linewidth and the residual TTF-TCNQ linewidth increase with irradiation dose (while the observed TTF-TCNQ linewidth decreases with the dose) and have roughly the same temperature-dependence (Fig. 5). Qn(TCNQ) $)_{2}$ is probably one of the most one-dimensional organic conductors $\left(\sigma_{\|} / \sigma_{\perp} \simeq 2500\right)$ [25], so the transverse hopping frequency and the associated spin relaxation are extremely low. It is not surprising then within the framework of our decomposition assumption, that the residual linewidth of irradiated TTF-TCNQ behaves similarly to the irradiated $\mathrm{Qn}(\mathrm{TCNQ})_{2}$ linewidth. Now studies of spin relaxation in pure and disordered $\mathrm{Qn}(\mathrm{TCNQ})_{2}$ at low and very low temperatures emphasize the following points [24, 26-28]. Hyperfine interaction is probably less efficient than dipolar interaction between the spins localized on adjacent segments [27, 29]. Irradiation generates spins with a smaller spatial separation, i.e., larger dipolar broadening [24]. A simple calculation gives this broadening proportional to the defect concentration [26]. The introduction of the exchange interaction describes the increase of the $\mathrm{Qn}(\mathrm{TCNQ})_{2}$ linewidth on cooling below $10 \mathrm{~K}$ [27]. At higher temperatures motional narrowing is more efficient and increases also with temperature. Anyhow both narrowings are sufficiently large to give a single Lorentzian line in the whole $T$ - and $c$-range.

The small temperature dependence of the coefficients $\alpha$ and $\beta$ (see Table I) is related to the competition between two types of narrowing. The possibility of a crossover between localized and mobile electrons has been demonstrated by nuclear spin-lattice relaxation measurements in $\mathrm{Qn}(\mathrm{TCNQ})_{2}$ [30]. At high temperatures the results are consistent with onedimensional diffusion with hopping between segments (or 1-D diffusion with random barriers), while below $20 \mathrm{~K}$ the localization is complete with a local spin correlation function described by disordered Heisenberg antiferromagnetism.

\section{Conclusion.}

We demonstrate that temperature and defect concentration dependence of the ESR linewidth for disordered organic conductors results of the association of two mechanisms.

The first one which narrows the signal comes from an increase of the spin-lattice relaxation time when electrons are more localized on segments. The second one which consists in a linear increase of the linewidth comes from the interaction between localized spins. To describe the latter contribution, one has to take into account, depending on the temperature, the dipolar and/or hyperfine interaction as well as exchange and/or motional narrowing. These results developed for the TTF-TCNQ case could be generalized to many other organic conductors.

On the other hand, below $100 \mathrm{~K}$ and under irradiation, we observe for TTF-TCNQ a crossover between bi- and one-dimensional types of electronic response to disorder. The microscopic dimensionality governs the ESR linewidth, and when the interchain motion is diffusive the linewidth is proportional to the transverse frequency of jump.

\section{Acknowledgments.}

We wish to thank F. Beuneu and L. Zuppiroli for numerous and fruitful discussions throughout the development of this paper. We are indebted to J. M. Fabre and K. Bechgaard for high quality crystals. 


\section{References}

[1] Tomkiewicz, Y., Phys. Rev. B 19 (1979) 4038.

[2] Flandrois, S., Coulon, C., Delhaes, P., Chasseau, D., Hauw, C., Gaultier, J., Fabre, J. M., Giral, L., Mol. Cryst. Liq. Cryst. 79 (1982) 307.

[3] Carneiro, K., Scott, J. C., Engler, E. M., Solid State Commun. 50 (1984) 477.

[4] Tomkiewicz, Y., Engler, E. M., Schultz, T. D., Phys. Rev. Lett. 35 (1975) 456.

[5] Coulon, C., Delhaes, P., Amiell, J., Manceau, J. P., Fabre, J. M., Giral, L., J. Physique 43 (1982) 1721.

[6] Pedersen, H. J., Scott, J. C., BechgaArd, K., Phys. Rev. B 24 (1981) 5014.

[7] Sanquer, M., Thèse d'Université Paris-Sud (1985) CEA-R-5335.

[8] Korin-Hamzic, B., Miljak, M., Cooper, J. R., in Proceedings of the International Conference on Low-dimensional conductors, Boulder, Colorado (August 1980) published in Mol. Cryst. Liq. Cryst. 85 (1982) 177.

[9] Mihaly, G., Zuppiroli, L., Philos. Mag. A 45 (1978) 549.

[10] Beuneu, F., Monod, P., Phys. Rev. B 18 (1978) 2422.

[11] Delmaes, P., Manceau, J. P., Guerard, D., Synthetic Metals 2 (1980) 277.

[12] Overhauser, A. W., Phys. Rev. 89 (1953) 689.

[13] Elliott, R. J., Phys. Rev. 96 (1954) 266.

[14] YAFET, Y., Solid State Physics, V. 14, edited by H. Ehrenreich, F. Seitz, D. Turnbull (Academic, N.Y.) 1965.

[15] Tomkiewicz, Y., Taranko, A. R., Phys. Rev. B 18 (1978) 733.

[16] Weger, M., J. Physique Colloq. 39 (1978) C6-1456.

[17] Soda, G., Jerome, D., Weger, M., Alizon, J., Gal-
LICE, J., Robert, H., FABre, J. M., Giral, L., J. Physique 38 (1977) 931.

[18] BLoch, A. N., in Proceedings of the International Conference on Organic Conductors and Semiconductors, Siofok (1976) published in Lecture Notes in Physics 65 (1976) 317.

[19] Zuppiroli, L., Bouffard, S., BechgaARD, K., Hilti, B., Mayer, C., Phys. Rev. B 22 (1980) 6035.

[20] ZuPPIROLI, L., Proceedings of the Conference « Experiments on Clusters » Königstein, October 1983. Berichte der Bunsen-Gesellschaft für physikalisch Chemic (1984) 304.

[21] Forro, L., Bouffard, S., Zuppiroli, L., J. Physique Colloq. 44 (1982) C3-927.

[22] Kawabata, A., J. Phys. Soc. Japan 29 (1970) 902. TAUPIN, C., J. Chem. Solids 28 (1967) 41.

[23] Thouless, D. J., Phys. Rep. 13 (1974) 93.

[24] Sanny, J., Grüner, G., Clark, W. G., Solid State Commun. 35 (1980) 657.

[25] DevreuX, F., Holczer, K., Nechtschein, M., Minier, M., J. Physique 44 (1983) 331.

[26] Sanny, J., Clark, W. G., Solid State Commun. 35 (1980) 473.

[27] Soos, Z. G., Bondeson, S. R., Proceedings of the International Conference on Low-dimensional conductors, Boulder, Colorado (August 1980) published in Mol. Cryst. Liq. Cryst. 85 (1982) 19.

[28] Devreux, F., Nechtschein, M., Grüner, G., Phys. Rev. Lett. 45 (1980) 53.

[29] Devreux, F., Jeandey, C., Nechtschein, M., Fabre, J. M., Giral, L., J. Physique 40 (1979) 671.

[30] Clark, W. G., Glover, K., Law, M. D., Azevedo, L. J., J. Physique Colloq. 44 (1980) C3-1493. 